\title{
Sarcoidosis Presenting as a Case of Pulmonary Thrombotic Disease- An Unusual Presentation
}

\author{
Muhammad Haseeb ul Rasool, MBBS ${ }^{1^{*}}$ (D), Sara Z Khan, MD² (i) and Pervaiz lqbal, MD, FCCP (iD) \\ ${ }^{1}$ King Edward Medical University, Pakistan \\ ${ }^{2}$ American University of Antigua, Antigua and Barbuda \\ ${ }^{3}$ Kings Brook Jewish Medical Center, USA
}

*Corresponding author: Muhammad Haseeb ul Rasool, MBBS, King Edward Medical University, Lahore, Pakistan, Tel: $+1-(609)-721-5736$

\begin{abstract}
Sarcoidosis is a chronic inflammatory disease involving multiple organ systems for which the inciting stimulus is unknown. It is characterized by elevated markers of inflammation and disseminated granuloma in the affected organs. Factor $\mathrm{V}$ Leiden deficiency has been long associated with an elevated risk of thrombosis, however, it has been observed in the patients having sarcoidosis, a secondary inciting stimulus is required to trigger the thromboembolic phenomenon, as to date only a few cases of Primary thrombosis have been reported in Factor $\mathrm{V}$ Leiden deficient patients culminating in an increased incidence of thromboembolic disease. We are presenting a case of a patient presenting with cerebrovascular events and pulmonary embolism, who was diagnosed later diagnosed to have sarcoidosis with an associated Protein S deficiency and factor $\mathrm{V}$ Leiden mutation making her predisposed to thromboembolic disease. It has been speculated that steroids as a treatment elevate the risk of thrombosis in such patients instead of the inflammatory process per se. however; this patient experienced a thrombotic episode before the start of medications. Additionally, an anatomical relationship has been reported in the literature between the site of sarcoidosis and the development of thrombosis, but the patient had repeated episodes of cerebrovascular events, despite no evidence of involvement of cranial vasculature or any heart defect, that could cause paradoxical embolization. This case report can help to elicit the relation of sarcoidosis with the increased tendency towards thromboembolic disease.
\end{abstract}

\section{Introduction}

Sarcoidosis is a chronic inflammatory condition characterized by multiple organ involvement. It was first described by Professor Caeser Peter Moller Boeck, who initially identified the disease based on the skin lesion and later on confirmed the multiorgan involvement. To date, no specific inciting agent has been identified that causes Sarcoidosis. Sarcoidosis is characterized as a chronic granulomatous disease in the organ involved with macrophages and monocytes being the hallmark of the disease. Active disease simultaneously remits over years; however active disease has been associated with increased incidence of loco-regional venous thromboembolism and vascular stenosis.

\section{Case Details}

We present a case of a 41-year-old Female who presented to pulmonology clinic for management of pulmonary embolism. Ten days before the presentation, she took a flight from New York City to Miami for an abdominal liposuction procedure. During the preparation for surgery, the patient experienced sudden onset of fever, cough, and shortness of breath. Examination revealed bilateral crepitation with bilateral absent breath sounds at bases. Chest X-ray showed pleural effusion, so with suspicion of pneumonia with parapneumonic effusion, she was started on coamoxiclav and symptomatic care, and surgery was deferred. The following day, the patient's SOB, and chest pain worsened, and she started to experience weakness in her right arm, for which in the emergency department, a CT chest was performed. CT scan showed right-sided pneumonia with a single filling defect in 
Table 1: Pulmonary function tests.

\begin{tabular}{|c|c|c|c|c|c|c|c|}
\hline $\mathrm{Sr}$ & Parameter & Reference & $\begin{array}{l}\text { Pre-Dilatation } \\
\text { Measure }\end{array}$ & $\begin{array}{l}\text { Pre-Dilatation } \\
\text { Percentage } \\
\text { Reference }\end{array}$ & $\begin{array}{l}\text { Post-Dilatation } \\
\text { Measure }\end{array}$ & $\begin{array}{l}\text { Post-Dilatation } \\
\text { Percentage } \\
\text { Reference }\end{array}$ & $\begin{array}{l}\text { Post-Dilatation } \\
\text { Percentage } \\
\text { Change }\end{array}$ \\
\hline 1 & FVC & 3.07 & 2.31 & 75 & 2.54 & 83 & 10 \\
\hline 2 & FEV1 & 2.6 & 2.20 & 84 & 2.30 & 88 & 5 \\
\hline 3 & FEV1/FVC & 85 & 95 & & 91 & & \\
\hline 4 & FEF $25-75 \%$ & 3.02 & 2.95 & 98 & 2.74 & 91 & -7 \\
\hline 5 & PEF & 5.94 & 5.64 & 95 & 5.95 & 100 & 6 \\
\hline 6 & FET 100\% & & 2.31 & & 4.18 & & 81 \\
\hline 7 & VC & 3.07 & 2.31 & 95 & 2.54 & 83 & \\
\hline 8 & TLC & 4.64 & & & & & \\
\hline 9 & RV & 1.52 & & & & & \\
\hline 10 & RV/TLC & 33 & & & & & \\
\hline 11 & FRC & 2.40 & & & & & \\
\hline 12 & DLCO & 22.5 & 17.2 & 76 & & & \\
\hline
\end{tabular}

FVC: Forced Vital Capacity; FEV1: Forced Expiratory Volume in 1 second; FEF 25-75\%: Forced Expiratory Flow over Middle One Half of the FVC; PEF: Peak Expiratory Flow; FET 100: Forced Expiratory Time; VC: Vital Capacity; TLC: Total Lung Capacity; RV: Residual Volume; FRC: Functional Residual Capacity; DLCO: Diffusion Capacity of the Lung for Carbon monoxide

the right pulmonary artery. CT angiogram of head and neck showed patent anterior and posterior circulation. The patient was treated initially with Lovenox and symptomatic care which stabilized her condition, and later she was switched to Eliquis. A week later, during her return flight to New York City, she started to experience headache, slurring of speech, along with right arm weakness, that resolved in an hour. In $E D$, no source of thrombosis could be found, lower extremity Venous Doppler could not elicit any evidence of DVT. She admitted to having shortness of breath with exertion, associated with dull chest pain, but denied any fever, hemoptysis, leg swelling, and palpitation. The patient's history was significant for a single episode of deviation of angle of the mouth, headache, and slurring of speech, a year before the recent presentation, for which she received Alteplase at that incidence, which alleviated her symptoms. Despite extensive workup, no source of thromboembolic phenomenon could be traced. Slurring of speech improved over time, but she had continued to experience waxing and waning fatigue, shortness of breath, and headaches. A repeat CT chest showed multiple nodules in the right lung parenchyma, along with a filling defect in the right pulmonary artery, Left lung was sparsely involved except for old basilar atelectasis or scarring. The patient had no history of smoking or a family history of thrombotic capacity. Examination revealed bilateral normal pulmonary auscultation with equal and comparable air entry. Neurological examination was nonfocal and muscle power was equal in all four limbs (Table 1).

The patient's exercise capacity was within normal limits. Basic metabolic profile, EKG, and LFTs were within normal limits (Table 2).

CT chest, abdomen, and pelvis showed multifocal nodular lung infiltrates, right upper lobe predominant with peripheral confluence/consolidation in conjunction with multi-station lymphadenopathy, worse in the mediastinum/hila raising the strong possibility of sarcoidosis, for which tissue sampling was advised. Borderline cardiomegaly with patent central, main, and segmental pulmonary artery lacking any evidence of acute or chronic pulmonary embolism. The spleen was normal in size with several indeterminate lesions raising the possibility of sarcoidosis. Several indeterminate hepatic lesions were found which could be hemangiomata, for which contrast-enhanced MRI was suggested. It was suggested that alternatively, these findings could be due to lymphoma or metastatic disease of indeterminate primary source. But no evidence of lymphoma or primary tumor could be found on $C B C$, mammogram, abdominal or pelvic imaging. MRI confirmed hepatic hemangiomata, splenic lesions. Bronchoscopic biopsy of the lesion from the right lung showed findings consistent with sarcoidosis. Based upon the radiological evidence and Siltzbach classification system, the patient was having stage II disease and was started on albuterol for symptomatic SOB, and steroid for sarcoidosis control. Eliquis was continued with all the precautions for falls. Referral to hematologist was provided for evaluation and assessment of low Protein $S$ activity. Further plan for a follow-up assessment after 6 months to document disease status was planned (Figure 1, Figure 2, Figure 3 and Figure 4).

\section{Discussion}

Incidence of sarcoidosis among the US population varies between $5-40$ cases per 100,000, being more prevalent in Patients from African American ethnicity having 34 cases per 100,000 population [1]. Crawshaw $A P$, et al. elicited that patients with sarcoidosis had an 
Table 2: Lab results for procoagulant status.

\begin{tabular}{|c|c|c|c|}
\hline Sr & Test name & Value & Normal value \\
\hline 1 & Sickle cell screen & Negative & Negative \\
\hline 2 & White cell count & $7.7 \times 10^{3} / \mathrm{uL}$ & $4.0-11.0 \times 10^{3} / \mathrm{uL}$ \\
\hline 3 & Red cell count & $4.4 \times 10^{6} / \mathrm{uL}$ & $3.9-5.0 \times 10^{6} / u L$ \\
\hline 4 & Hemiglobin & $11.4 \mathrm{~g} / \mathrm{dL}$ & $11.9-15.5 \mathrm{~g} / \mathrm{dL}$ \\
\hline 5 & Hematocrit & $35.6 \%$ & $35-45 \%$ \\
\hline 6 & Mean corpuscular hemoglobin & $26 \mathrm{pg}$ & $25-33 \mathrm{pg}$ \\
\hline 7 & Mean corpuscular volume & $80.9 \mathrm{fL}$ & 80-99 fL \\
\hline 8 & Platelets & $377 \times 10^{3} / \mathrm{uL}$ & $150-450 \times 10^{3} / \mathrm{uL}$ \\
\hline 9 & Protein S functional & 28 & $63-140$ \\
\hline 10 & Factor V leiden & $\begin{array}{l}\text { FVHET2 } \\
\text { (Single R506Q mutation identified- } \\
\text { heterozygte) }\end{array}$ & \\
\hline 11 & Protein $\mathrm{C}$ functional & 112 & $73-180$ \\
\hline 12 & Factor $\mathrm{V}$ activity & 78 & $70-150$ \\
\hline 13 & Anti-cardiolipin antibodies IgG, Quantitative & $<9$ & $0-14$ \\
\hline 14 & Anti-cardiolipin antibodies IgM, Quantitative & 13 & $0-12$ \\
\hline 15 & Anti-cardiolipin antibodies IgA, Quantitative & $<9$ & $0-11$ \\
\hline 16 & Beta-2-glycoprotein I antibodies, IgG & $<9$ & $0-20$ \\
\hline 17 & Beta-2-glycoprotein I antibodies, IgA & $<9$ & $0-25$ \\
\hline 18 & Beta-2-glycoprotein I antibodies, IgM & $<9$ & $0-32$ \\
\hline 19 & Antiphosphatidylserine $\lg \mathrm{M}$ & 16 & $0-25$ \\
\hline 20 & Antiphosphatidylserine $\lg A$ & 03 & $0-20$ \\
\hline 21 & Antiphosphatidylserine lgG & 02 & $0-11$ \\
\hline 22 & Prothrombin time & 14.2 seconds & 10-14 Seconds \\
\hline 23 & International normalized ratio & 1.2 & $0.8-1.1$ \\
\hline 24 & Partial thromboplastin time & 34 seconds & $21-35$ \\
\hline
\end{tabular}

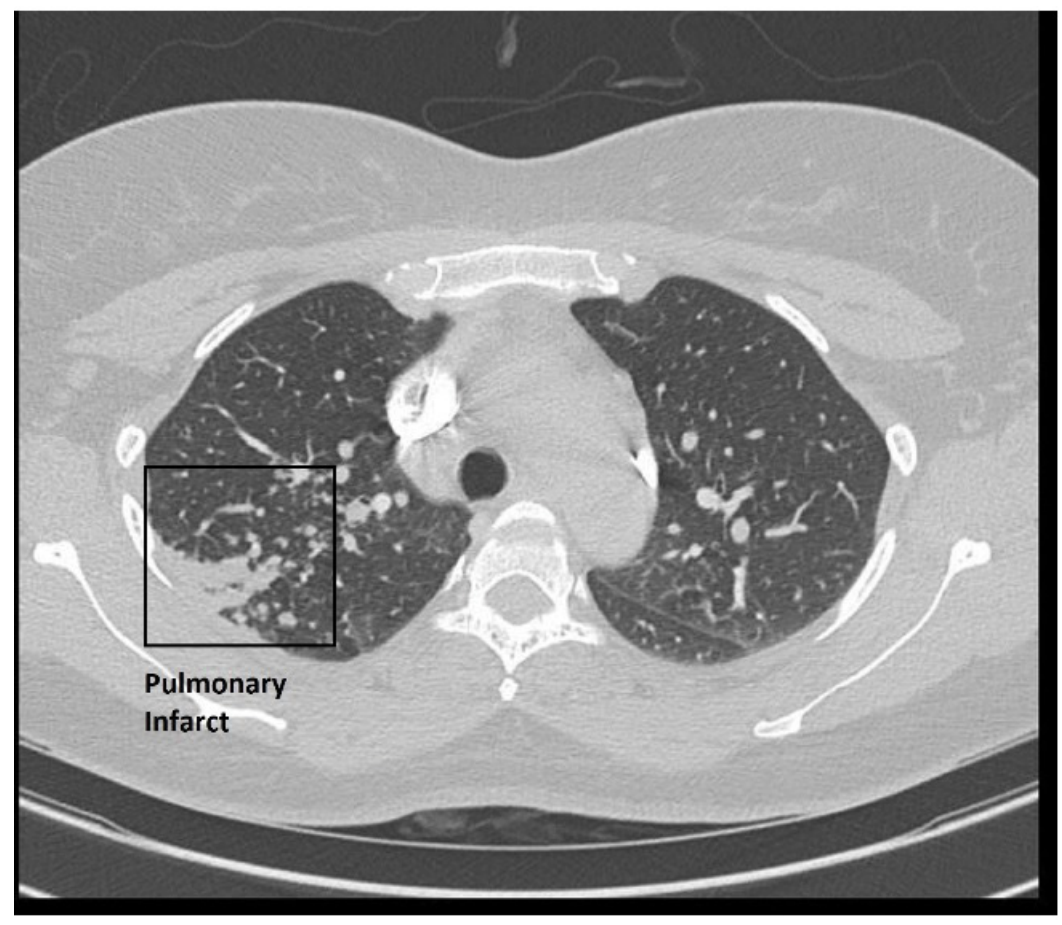

Figure 1: CT chest showing pulmonary infarct. 


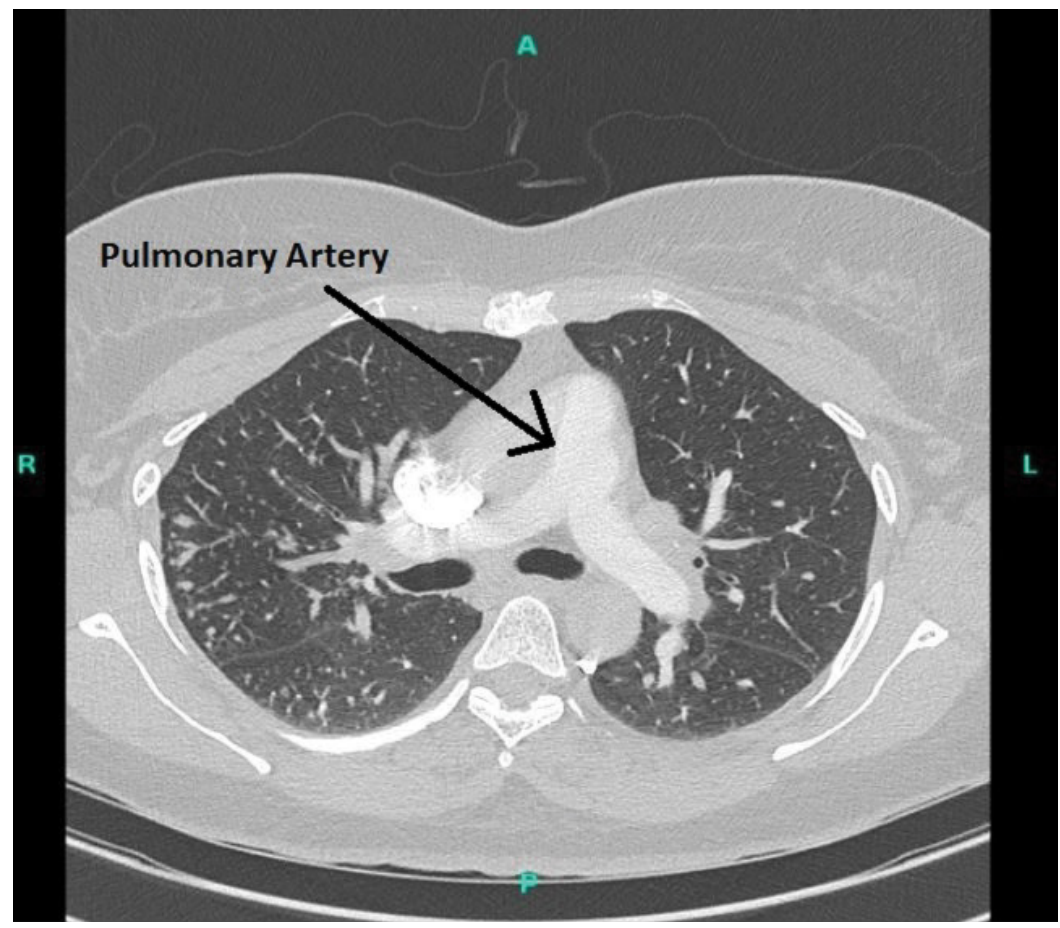

Figure 2: CT chest showing pulmonary artery.

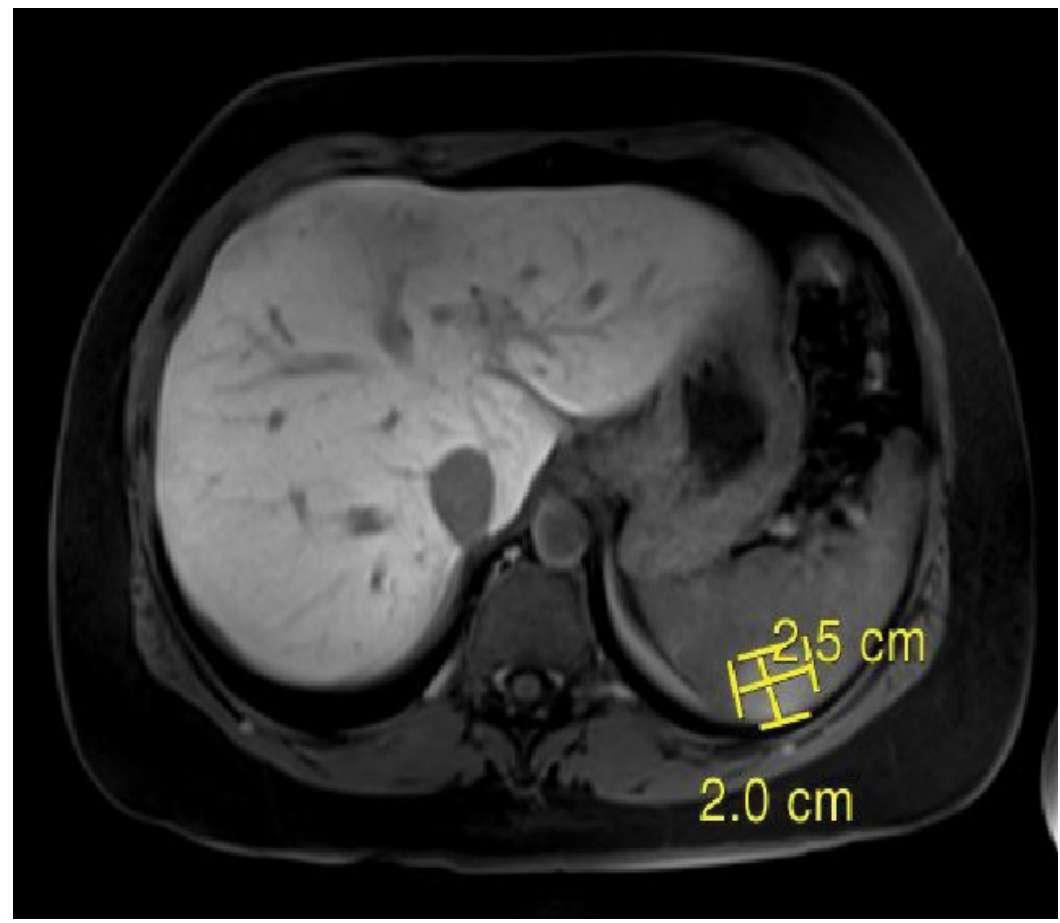

Figure 3: MRI abdomen showing splenic granuloma (Axial section).

increased risk of Pulmonary Embolism (Rate Ratio 2.0, $95 \% \mathrm{Cl}: 1.1$ to 3.4 , under age of 65 years) [2]. A similar risk for PTE was found by Swigris and colleagues [3]. Nevertheless, it has not been established whether sarcoidosis is a risk factor for thromboembolic disease. In literature, an anatomical relationship between the incidence of venous thromboembolic disease and the site of active sarcoidosis has been described, exemplified by mural thrombosis with myocardial sarcoidosis [4], cerebral vein thrombosis with neurosarcoidosis $[5,6]$, thoracic vein thrombosis with mediastinal sarcoidosis [7], and Portal vein thrombosis in Hepatic Sarcoidosis [8]. Factor V Leiden mutation has been long affiliated with increased risk of thrombophilia, however, studies have shown that these individuals, even though tend to thrombophilia, need a second acquired stimulus to develop a clinically significant thrombotic event [9]. Smoking has also failed to show a significant risk of association with pulmonary thromboembolism in patients having sarcoidosis [10]. 


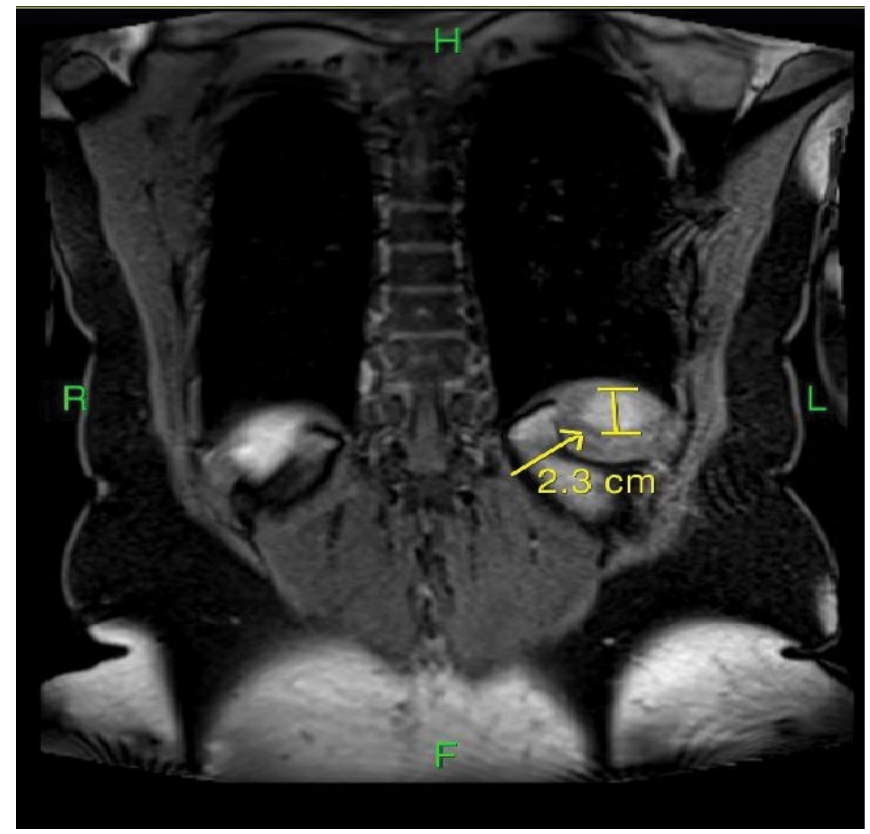

Figure 4: MRI abdomen showing splenic granuloma (Coronal section).

Kolluri, et al. in a retrospective study found that with multivariate regression analysis, the steroid therapy for the treatment of sarcoidosis, rather than the inflammatory disease process, were the most significant risk factor for pulmonary thromboembolism $(H R=$ 3.12, $p=0.007$ ) [11]. There have been various other studies where anti-inflammatory therapy was found to be the actual risk for thromboembolic disease rather than the inflammatory process itself. Tandon, et al. reported a case series of seven cases, where sarcoidosis presented with pulmonary hypertension due to chronic thromboembolic phenomenon, however, no evidence of pulmonary hypertension could be found in this patient [12].

\section{Conclusion}

The patient had experienced repeated episodes of thrombosis involving cranial vasculature, but her investigations showed only weak evidence of related deficiencies. Even though the patient was heterozygous for Factor V Leiden, but had normal Protein C activity. Prolonged air travel could be a possible inciting factor for the thrombotic event, but there was no evidence of deep vein thrombosis on imaging. The patient's presentation of waxing and waning headaches and right arm weakness can be explained by transient Ischemic attacks. Sarcoidosis was the only clinical risk factor to explain the patient's propensity to have thrombotic events. The patient had thrombotic episodes before the start of the treatment which contradicts the finding of steroids being the risk factor for thrombosis instead of the inflammatory process.

\section{References}

1. Kamangar N, Mosenifar Z (2020) What is the incidence of sarcoidosis in the US? MedScape.
2. Crawshaw AP, Wotton CJ, Yeates DGR, Goldacre MJ, Ho LP (2011) Evidence for association between sarcoidosis and pulmonary embolism from 35-year record linkage study. Thorax 66: 447-448.

3. Jeffrey JS, Amy LO, Tristan JH, Evans RFP, Joshua JS, et al. (2011) Increased risk of pulmonary embolism among US decedents with sarcoidosis from 1988 to 2007 . Chest 140: 1261-1266.

4. Wynne JW, Ryerson GG, Dalovisio J (1979) Myocardial sarcoidosis complicated by mural thrombosis. Thorax 34 : 127-129.

5. Akova YA, Kansu T, Duman S (1993) Pseudotumorcerebri secondary to dural sinus thrombosis in neurosarcoidosis. $J$ Clin Neuroophthalmol 13: 188-189.

6. Selvi A, Diakou M, Giannopoulos S, Zikou AK, Argyropoulou $\mathrm{Ml}$, et al. (2009) Cerebral venous thrombosis in a patient with sarcoidosis. Intern Med 48: 723-725.

7. Marc K, Bourkadi JE, Beamor J, Iraqi G (2008) Thoracic venous thrombosis in the course of sarcoidosis. Rev Mal Respir 5: 105-106.

8. Moreno Merlo F, Wanless IR, Shimamatsu K, Sherman M, Greig P, et al. (1997) The role of granulomatous phlebitis and thrombosis in the pathogenesis of cirrhosis and portal hypertension in sarcoidosis. Hepatology 26: 554-560.

9. Goljan Geremek A, Geremek M, Puscinska E, Sliwinski $P$ (2015) Venous thromboembolism and sarcoidosis: Coincidence or coexistence? Cent Eur J Immunol 40: 477-480.

10. Vorselaars ADM, Snijder RJ, Grutters JC (2012) Increased number of pulmonary embolisms in sarcoidosis patients. Chest Journal 141: 826-827.

11. Kolluri N, Elwazir M, Rosenbaum A, Blauwet L, Abou Ezzeddine O, et al. (2020) Glucocorticoid therapy rather than the inflammatory state is associated with pulmonary embolism and deep vein thrombosis in cardiac sarcoid. EHJ 41.

12. Tandon R, Baughman RP, Stanley J, Khan AA (2017) The link between chronic thromboembolic pulmonary hypertension and sarcoidosis: Association or visual masquerade? Sarcoidosis Vasc Diffuse Lung Dis 34: 352355. 